\title{
CONNECTIVITy SERVICES FOR Automotive Mobility APPLiCATIONS
}

\author{
Juan José Pérez, Marcos Pillado and Abel Carbonell \\ Applus+ IDIADA \\ E-mails: juanjose.perez@idiada.com, marcos.pillado@idiada.com, acarbonell@idiada.com
}

\begin{abstract}
Cooperative and automated driving vehicles bring more complexity to the traditional development, test and validation procedures based on the V-Model where the vehicle is considered a complete system. Vehicle communication capabilities and its interaction with other road users introduce new requirements which need to be taken into account from the design stages. This paper presents the Connected Vehicle Lab at IDIADA, a comprehensive environment for the design and validation of connected and automated driving technologies. Built on top of the state-of-the-art proving grounds, a C-ITS network built upon ETSI ITS G5 compliant roadside units and a dedicated cellular network deployed with the latest technologies where the parameterization can be customized on demand, allows the recreation of any real-life driving situation in a fully controlled scenario, while ensuring all the safety, security and confidentiality requirements are met. This facility is meant to support the automotive stakeholders through the whole vehicle development cycle.
\end{abstract}

\section{INTRODUCTION}

On board sensors and cameras limit the information that a vehicle is able to receive and process to the nearest environment and line-of-sight applications. To further expand these information acquisition capabilities with the aim of increasing traffic efficiency and safety, Cooperative Intelligent Transport Systems (C-ITS) technologies rely on information sharing between vehicles by means of wireless communication systems and networks. Furthermore, vehicle connectivity will be a key enabler for higher levels of automation to be implemented. With Cellular Vehicle to Everything (C-V2X) and 802.11p as the current available standards for the lower layers (PHY and MAC), newer approaches have to be considered to include the assessment of these additional elements as part of the validation process. This paper presents IDIADA's vision on how to address this challenge by introducing an innovative wireless communication network solution.

\section{BACKGROUND AND MOTIVATION}

IDIADA's development process complies with the V-Model showed in Figure 1 which is based on the ISO26262. IDIADA follows this process oriented development methodology to minimize risks, development time and ensure a level of quality. Focussing on the testing part of V-Model (right), the testing of cooperative systems increases the complexity compared to testing traditional automotive systems, the main reason behind is that cooperative systems interact with other ITS systems (vehicles, pedestrians or roadside infrastructure). For this purpose, IDIADA has built a connected vehicle testing platform equipped with the latest cellular communications technologies and ETSI ITS G5 compliance in order to facilitate the building of evermore reliable systems by offering its facilities and tailoring the platform to the 
particular test scenario needs, in order to repeatedly conducting tests and evaluations that are difficult or even impossible on public roads due to constantly changing road environments.

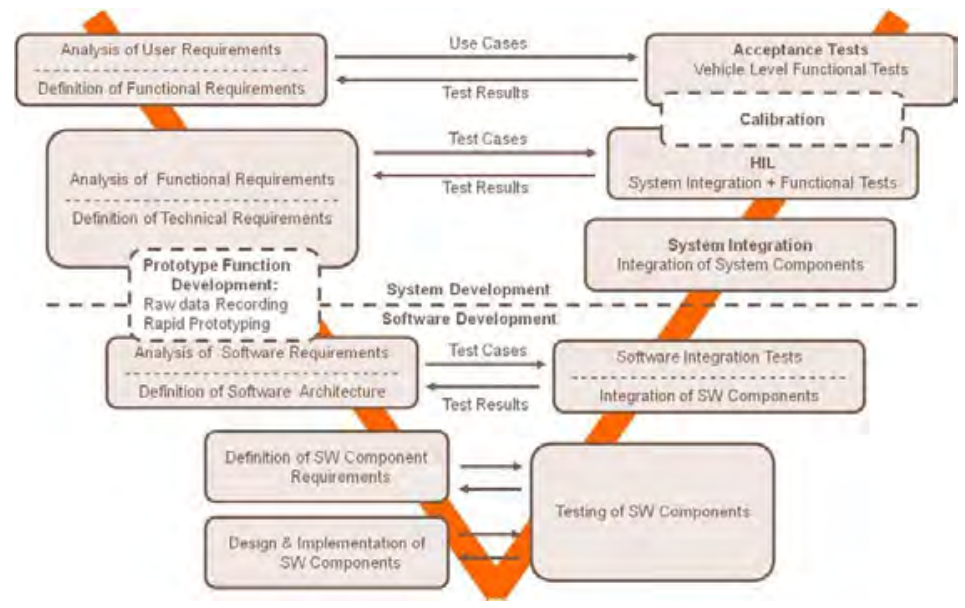

Figure 1 - V-Model

In particular, at system level, IDIADA considers of special relevance the following testing activities for connected and automated driving:

- Conformance testing, the process for testing that an implementation is compliant with a protocol standard; it is realized by test systems simulating the protocol with test scripts executed against the implementation under test (IUT).

- Interoperability testing, the analysis of compatibility issues where vendors had followed the standards, but their interpretation of the requirement differed. The results guarantee the capability for distributed components of a system to communicate successfully and to exchange consistent data.

- Performance assessment, analysis of necessary minimum levels of performance for deploying services, in particular related to antenna performance and GNSS positioning performance.

- Vehicle Integration Testing, Electromagnetic Compatibility (EMC) test assures the proper operation of different equipment operating in the same electromagnetic environment without causing interference and without suffering from other equipment electromagnetic radiation (immunity).

- Functional Testing, beyond capabilities relating to interoperability and performance, cooperative ITS devices need also to provide suitable functional behaviours. Therefore functional requirements are necessary to be fulfilled by all devices forming the cooperative systems. Functional requirements need to focus on the procedures beyond the communication and exchange of data. For instance functional requirements shall cover the performance at the application level, ensuring the warnings received by ITS station are leading to display appropriate and timely warning to the driver. Therefore ensuring the reliability of the C-ITS application concerns the whole system, in extension to the combination of the different C-ITS devices. Functional field testing would assess appropriate performances of the C-ITS services, at the application and system level, rather than at the device level. Evaluation of the cooperative ITS system performance in real driving conditions, including both closed test tracks and public roads, are expected to assess complementary features, being essential for the successful execution of the cooperative applications. Therefore these validation procedures enable assessing the performances of the complete systems, including vehicle, roadside units, ITS Central Stations and the cellular network. The procedures 
are therefore not assessing performance of isolated devices but the performances of the whole system, while giving the opportunity to identify which device is responsible for faulty behaviour. The tests must be primarily performed on closed test tracks, equipped with cooperative ITS equipment including crossings with traffic lights where conditions are controlled and repeatability is guaranteed. These two conditions are key to isolate the system in order to analyse its performance and identify issues that otherwise cannot be easily determined due to the randomness of performing tests on public roads.

\section{WIRELESS COMMUNICATIONS IN THE VEHICLE}

Most of today's vehicles are somehow connected to external application servers, networks and the internet by means of embedded communication modules, tethered solutions relying on the driver's smartphone or integrated solutions where the apps are directly deployed on the car's infotainment system [1]. With the advent of new standards like the Cellular Vehicle-toEverything (C-V2X) included in 3GPP Release 14 [2]-[3], which in its first version expands the LTE platform to provide Vehicle-to-Vehicle (V2V) direct communication capabilities, cellular networks are likely to become more and more relevant as a main technology enabler for the C-ITS technologies deployment and higher levels of automation implementation. On the other hand, $802.11 \mathrm{p}$ is already established as a well-proven standard specifically designed to meet every V2X application requirement and performance specification.

Mandatory regulation of vehicle emergency call systems also plays a key role in the adoption of in-vehicle communication devices. The Minimum Set of Data (MSD) including all the relevant information relating to a vehicle crash, i.e. vehicle identification, vehicle propulsion storage type, time stamp, vehicle location and direction, is sent in-band over a GSM or UMTS voice channel connection, thus requiring a cellular communication module to be installed in the vehicle. The Russian implementation of the emergency call service, ERA-GLONASS, became mandatory for all transportation vehicles released for free circulation in the Customs Union member states from January 1st 2017. The EU implementation named eCall will require all new cars to be equipped with this technology from March 31st 2018, and several other countries like Turkey and India are developing their own regulations based on the existing ones. NG 9-1-1 systems to be implemented in the US will allow sending text messages, pictures, videos and other information from any device, while Japan HelpNet system provides the GPS position combined with a preregistered car information database and will be further enhanced with statistical processing capabilities.

The progressive introduction of these wireless technologies in the vehicle will bring a wide variety of new cooperative and connected services and applications to be implemented on top of the lower layers standards, with its importance rapidly growing towards the future introduction of the fully autonomous vehicles. The development of such kind of services and applications requires extensive and cost-consuming testing efforts, thus revealing the need for new test beds and tools that ease the execution of these tests while granting safety and security at the same time. Traditional test tracks are suitable for traditional cars, but cooperative, connected and automated vehicles demand new test environments that can provide a controlled and fully configurable network covering both physical and digital infrastructure in order to be able to replicate and recreate network scenarios in a safe manner. 
For specific scenarios, one usually requires to drive many kilometres to find specific challenging conditions, which therefore implies involving a great number of resources. In some countries is not even possible to carry out certain tests in public roads, as it is forbidden by the current regulations. Moreover, commercial cellular networks are not suitable for this kind of tests since their working conditions are not under control: available capacity varies depending on the number of users connected and the usage they make of the network resources, coverage conditions cannot be modified and network availability may be affected by network operation and maintenance activities. For the testing results and subsequent conclusions to be reliable there is a need to control network parameters in order to replicate specific scenarios.

On the other hand, commercial networks are not promptly suited with the latest features and future solutions that are required for enabling efficiency and safety applications, as their implementation depends on the mobile network operator interest on offering those features and requires a deployment plan where the network size and the number of affected network elements must be taken into account. Last but not least, privacy issues may be avoided keeping sensitive data in-house, which is another benefit that a private environment provides.

\section{HETEROGENEOUS V2X NETWORKS}

Several research studies have been conducted comparing the performance of $802.11 \mathrm{p}$ and CV2X standards for Connected and Automated Vehicles applications [4]-[5]. While IEEE $802.11 \mathrm{p}$ standard was designed from the beginning to meet every V2X application requirement and it is ready for deployment after extensive field trials, the C-V2X standard introduced in 3GPP Release 14 enables V2V direct communication through the PC5 interface without the need of relying on the network as one of its working modes, potentially bringing superior performance in terms of latency, bandwidth and coverage range specially when taking into account the evolution path to $5 \mathrm{G}$, and at the same time taking advantage of an extensively deployed wireless network. However, the implementation of the 3GPP standard is in the initial trial stages and its application to commercial solutions will require some time.

Both standards specifications for PHY and MAC can be leveraged to support upper layers specifications from ETSI, ISO, SAE and IEEE, meaning that the same facilities and applications can be implemented on top of these lower layers.

These considerations bring a heterogeneous scenario where both standards will coexist as proposed in [6], with cooperative, connected and automated mobility development taking advantage of the benefits brought by both of them while minimizing the existing drawbacks. Even if the future evolution of their technical specifications takes one of them to dominate the market, or new contenders appears, current research works will dive in this interworking capabilities.

\section{CONNECTED VEHICLE LAB DESCRIPTION}

With the aim to replicate any possible network condition that a car may face while on the road, the capability to cover the test tracks with the whole set of access technologies allowing the development and implementation of C-ITS applications is a main requirement from the network design point of view. This includes both DSRC devices compliant with IEEE 1609 and ITS G5 standards and a cellular network covering all generations from $2 \mathrm{G}$ to $4 \mathrm{G}$, with the 
5G standard being under development at this stage. Although the first 5G standards will not be approved until March 2018, telecom vendors are already providing an evolution path for the current commercial networks to be updated while taking advantage of several benefits that can be implemented in the meantime.

\section{C-ITS Platform}

As a part of its wireless communication infrastructure, IDIADA has designed and deployed a C-ITS platform. IDIADA's proving ground is completely covered with ETSI ITS G5 and WiFi technologies. Topography information with $2 \mathrm{~cm}$ accuracy and D-GPS coverage is available too.

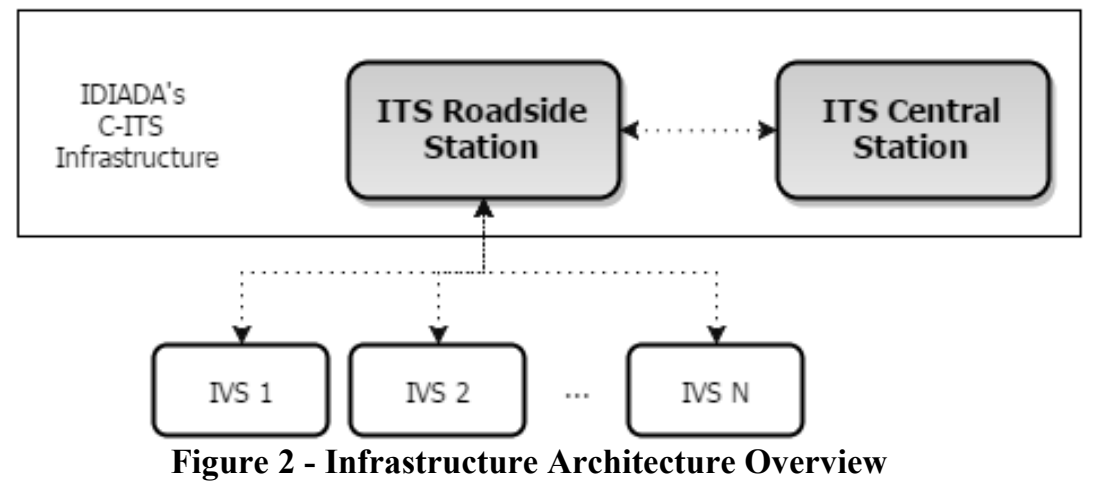

The components of the infrastructure deployed can be observed on Figure 2. A more detailed description of each component follows:

- ITS Roadside Station: ITS Roadside Stations (IRSs) are equipped with dual transceivers and strategically deployed along the High Speed Track (HST) to warranty 6 Mbps to the Dynamic Platforms and the HST under serious adverse circumstances (mobile IRSs can be easily deployed for the definition of specific scenarios). IRSs incorporate a Cohda MK5 radio [7], and their function is to collect the data provided by the IVSs and forward it to the ITS Central Station (ICS), as well as flexibly provide local applications (either standard based applications or new cooperative applications). The IRSs can be also used as an objective and independent system to observe the systems under test.

On Figure 3 is represented the position of the IRSs and its coverage. The C-ITS platform provides a minimum transfer rate of $6 \mathrm{Mbits} / \mathrm{s}$ under adverse conditions, which means robust links that consider all kind of negative encounters like bad weather conditions, wide fading margins, wide noise margins, consider Doppler Effect and coupling loss. Under more relaxed conditions, the C-ITS platform can cover $95 \%$ of IDIADA's test tracks and reach up to $21 \mathrm{Mbit} / \mathrm{s}$. 


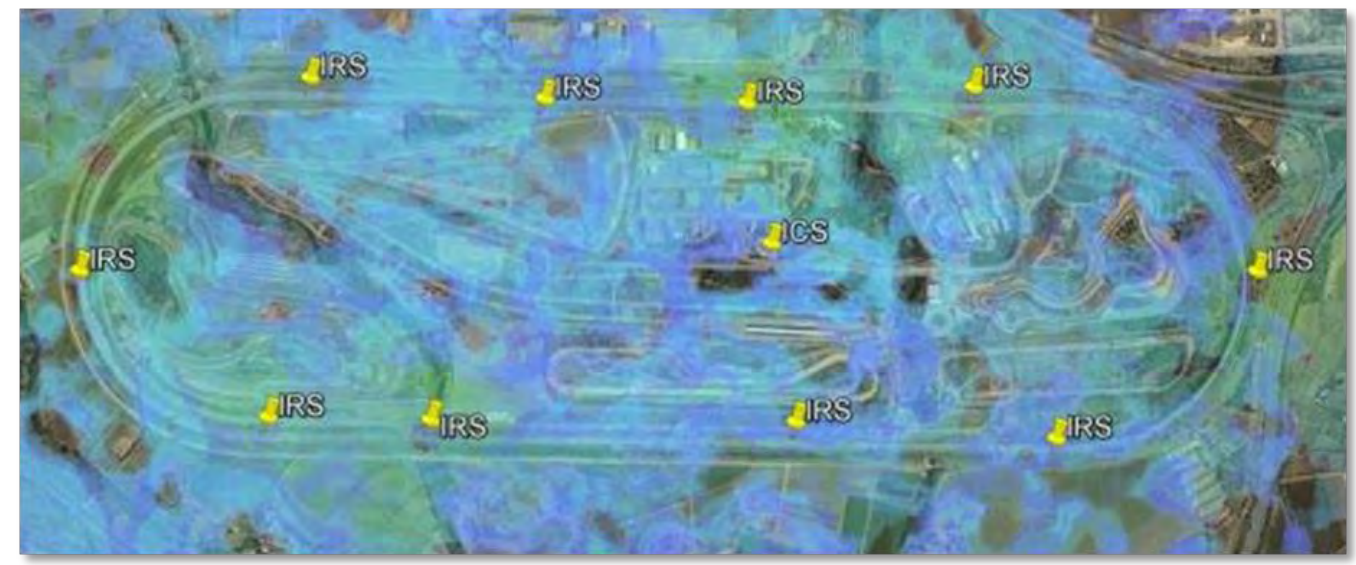

Figure 3 - Coverage Study

- ITS Control Station: All equipment is connected via high bandwidth links to the ICS that can monitor the network in real-time, track the vehicles and log the data for further analysis. For the purpose of providing a monitoring solution, the open source Nagios software [8] is used to monitor a total of 20 services. Nagios relies on SNMP agents, which are installed and configured on every IRS. These agents, upon receiving a query made by Nagios, supply a response with the data queried. For a more graphical representation of the monitored infrastructure, a Nagios extension called Nagvis [9] is used. Nagvis represents the monitored hosts in a map, see Figure 4. Last but not least, Nagiosgraph [10] provides an intuitive way of looking at historical data. This extension also uses a SQL database to retrieve monitored data.

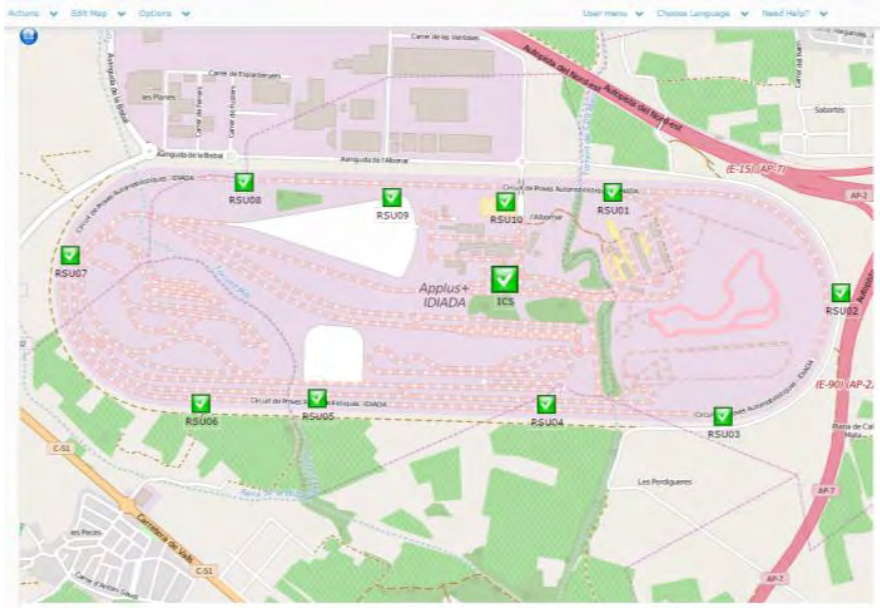

Figure 4 - Nagvis provides a geographical representation of the infrastructure

\section{Cellular network}

The other key asset of IDIADA's Connected Vehicle Lab is the cellular network deployed with the latest state-of-the-art technologies and prepared for the future evolution to 5G. Full coverage is provided to the proving ground by means of three multi-standard radio base stations, which complement the C-ITS infrastructure building together a comprehensive connectivity testing platform.

Cellular network can be separated into three main functional domains: Radio Access Network (RAN), Transport Network and Core Network (CN), all of them to be considered if a complete operating solution is to be deployed. Safety applications demand high performance 
networks in terms of bandwidth, latency, reliability and availability, requiring target values which are difficult or even not possible to reach by the current standards, requiring all the network elements and the interconnections between them to be carefully designed and implemented. A description of the adopted solution at IDIADA follows:

- Radio Access Network: the Radio Access Network connects the User Equipment (UE) through the air interface by implementing one or more radio technologies. The Connected Vehicle Lab include three macro base stations implementing 2G, $3 \mathrm{G}$ and $4 \mathrm{G}$ radio access technologies, configured with the latest available software releases and network features. As per the frequency spectrum usage, collaboration with leading mobile network operators grants permission to radiate in all the common frequency bands, allowing the setup of specific network scenarios where the coverage of a combination of these radio access technologies and frequency bands can be adjusted as desired. Figure 5 shows the location of the base stations within IDIADA's facilities:

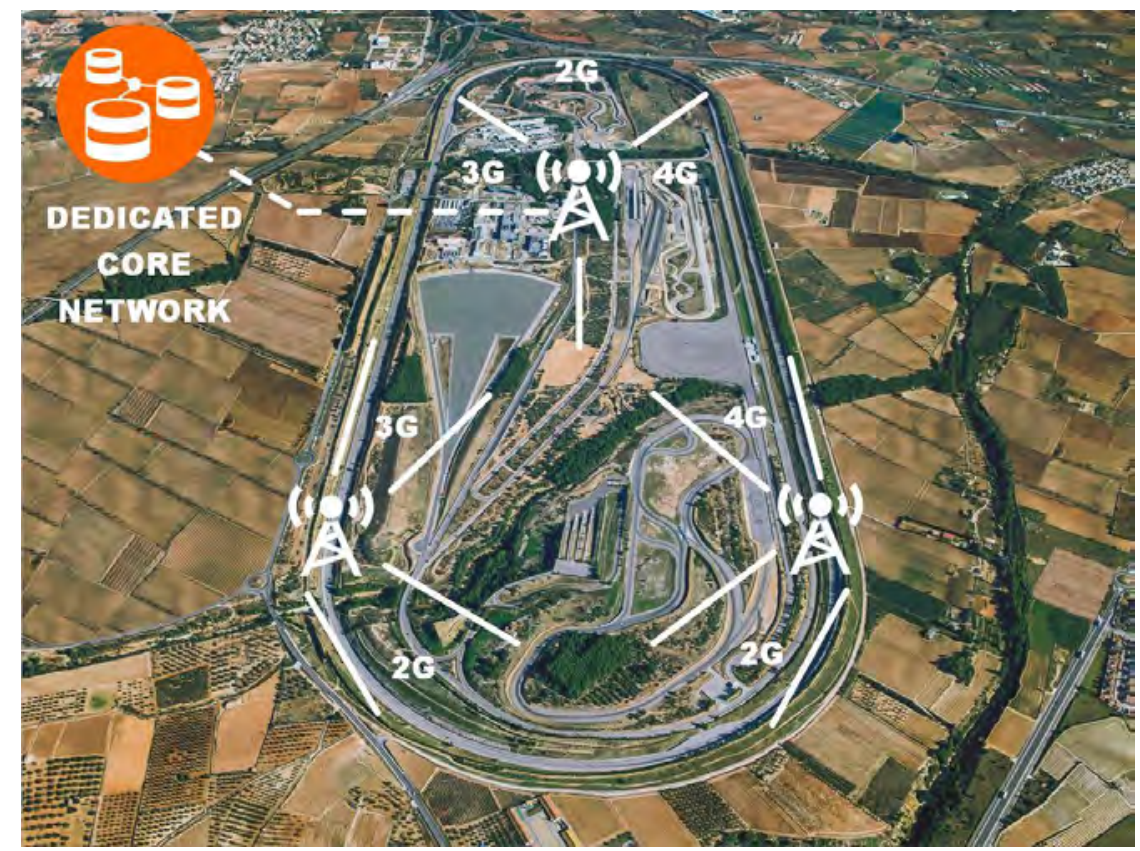

Figure 5 - Radio Base Station distribution

- Transport Network: The backhaul connecting the base stations to the Base Station Controller (BSC), the Radio Network Controller (RNC) and the Evolved Packet Core (EPC) is based on IP over optical fibre links, ensuring high reliability and high speed performance. With the aim to evaluate the impact of the transport solution in the network general performance, microwave and satellite backhaul inclusion as an alternative is under consideration. External Application Servers (AS) can be connected directly to the internal network or remotely using broadband connections, depending on the scope of the tests to be performed.

- Core Network: the Core Network performs the switching functions and provides connection to other external networks as the Public Switched Telephone Network (PSTN, Public Data Network (PDN) or Private Data Networks belonging to the users of the proving ground, among other messaging, authentication or charging functions. For $2 \mathrm{G}$ and $3 \mathrm{G}$ networks the Core is split into Circuit Switched (CS) and Packet Switched (PS) network elements, the former carrying voice services and the latter 
carrying data, while 4G is an entirely based PS network and even voice is an application implemented over the data layer known as VoLTE. Packet Switched (PS) Core Network elements carrying the network user traffic on the data plane are deployed locally in a dedicated server room.

The high-level network architecture is presented in Figure 6.

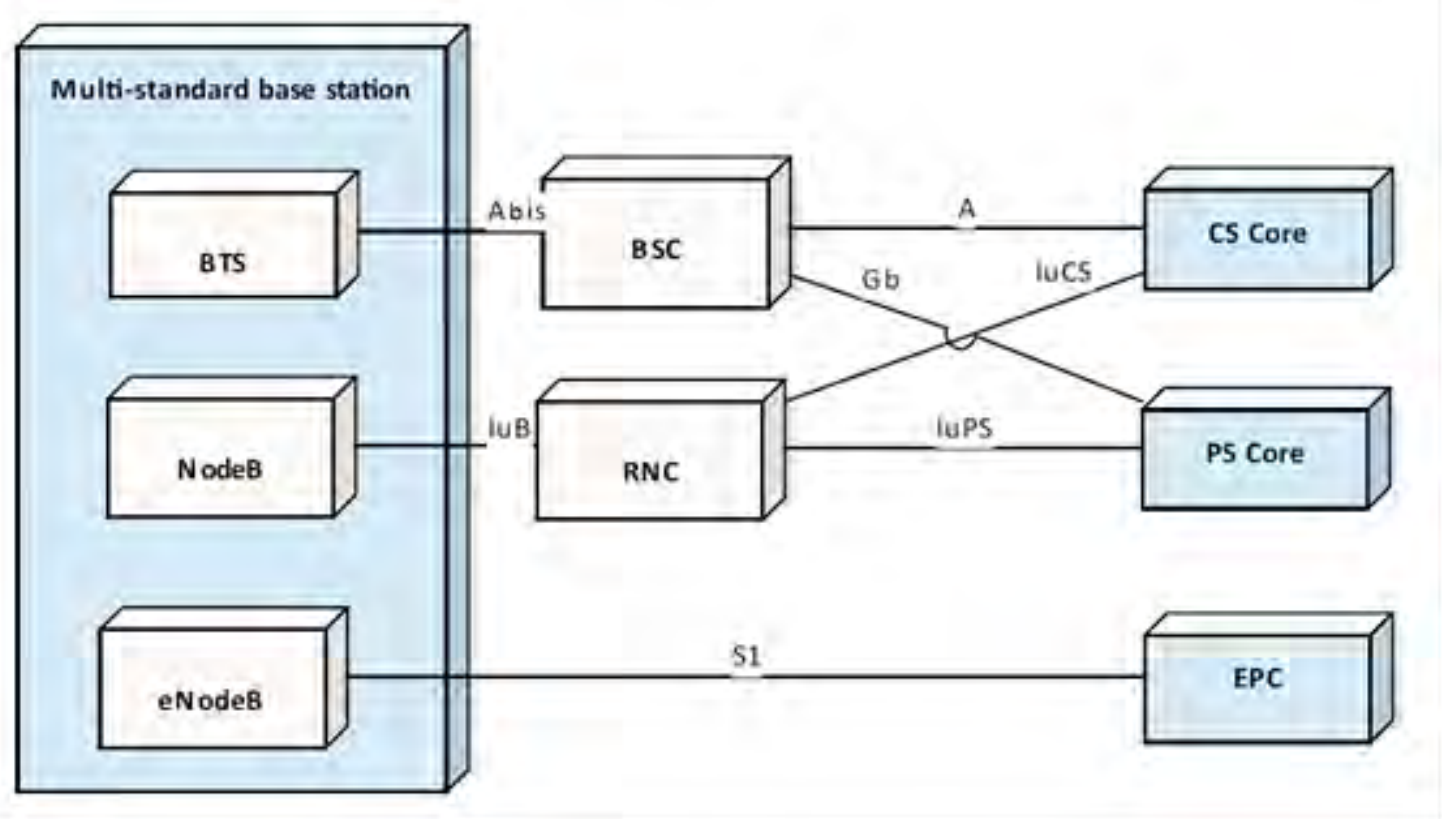

Figure 6 - Cellular Network High-Level Architecture

Current development onto $5 \mathrm{G}$ is for a high speed data network using multiple carriers and multiple spectrum bands which yield a higher efficiency of data per megahertz of spectrum. 3 GPP produces release levels of specifications and recommendations with the current version being R14 (Release 14), which is being rolled out over the current networks worldwide. The next release of new recommendations (R15) is planned for final ratification by September of 2018 after which a period of vendor development will take place with the first networks becoming available probably in early 2020. It is R15 which is generally referred to as $5 \mathrm{G}$.

Only by maintaining full compliance with critical 3GPP recommendations and specifications a high level of network development, interworking between elements and software platforms, evolution and security can be provided in the medium to long term. Moreover, to ensure that the developments on the proving ground can be rolled out commercially it is critical that the cellular network used closely matches what is available on public networks as all autonomous developments will need to use standard public providers without significant network modification. To that end, IDIADA's cellular network is built upon high-grade commercial equipment guaranteeing that relevant results are obtained.

\section{CONCLUSION}

The progressive introduction of wireless communication modules in the vehicle will foster the development of new cooperative, connected and automated mobility applications and services. This will require existing and new testing facilities to adapt its physical 
infrastructure to support the development of such kind of applications, embracing the wireless technologies meant to provide them with the necessary communication capabilities.

IDIADA's Connected Vehicle Lab implements both DSRC and cellular networks offering a comprehensive development and testing environment that will need to be validated in terms of its integration with C-ITS applications.

On the other hand, the continuous evolution of the network capabilities including hardware, software and new features to be implemented will be a main driver of the Connected Vehicle $\mathrm{Lab}$ in order to be able to match the commercial network characteristics with the aim of offering a real drive experience for development and testing purposes. Specifically, the network evolution towards $5 \mathrm{G}$ will be of the highest priority given the expected impact it will have in the development of a whole new set of connected vehicle applications, enabling at the same time an improvement on the performance of the existing ones and paving the way for full driving automation introduction.

\section{REFERENCES}

[1] GSM Association (GSMA) (2012), "2025 Every Car Connected: Forecasting the Growth and Opportunity (white paper)".

[2] 3GPP TR 36.885, Study on LTE-based V2X Services (Release 14), 3GPP Technical Specification Group Radio Access Network, v14.0.0, June 2016.

[3] 5G Automotive Association (5GAA) (2016), "The case for Cellular V2X for Safety and Cooperative Driving (white paper)", 8 p., November 2016 http://5gaa.org/wpcontent/uploads/ 2017/08/ 5GAA-whitepaper-23-Nov-2016.pdf

[4] A. Bazzi, B. M. Masini, A. Zanella and I. Thibault, "On the Performance of IEEE 802.11p and LTE-V2V for the Cooperative Awareness of Connected Vehicles," in IEEE Transactions on Vehicular Technology, vol. 66, no. 11, pp. 10419-10432, Nov. 2017.

[5] Blasco et. al, "3GPP LTE enhancements for V2V and comparison to IEEE 802.11p", 11 th ITS European Congress, Glasgow, Scotland, June 2016.

[6] K. Abboud, H. A. Omar and W. Zhuang, "Interworking of DSRC and Cellular Network Technologies for V2X Communications: A Survey," in IEEE Transactions on Vehicular Technology, vol. 65, no. 12, pp. 9457-9470, Dec. 2016.

[7] Cohda Wireless "MK5 V2X On-Board Unit" http://www.cohdawireless.com/solutions/ hardware/mk5-obu/

[8] Nagios Software IT Infrastructure Monitoring Solution. https://www.nagios.org/

[9] Nagvis, Nagios visualization addon. http://www.nagvis.org/

[10] Nagiosgraph, Nagios historical data visualization add-on.

http://nagiosgraph.sourceforge.net/ 